\title{
The Adipocerous Ossein- Chondroid Lipoma
}

\author{
Anubha Bajaj* \\ Histopathologist in A B Diagnostics, New Delhi, India \\ Submission: December 06, 2020; Published: December 16, 2020 \\ *Corresponding author: Anubha Bajaj, Histopathologist in A B Diagnostics, New Delhi, India
}

\section{Preface}

Mesenchymal soft tissue adipocytic neoplasms are frequently discerned tumors demonstrating diverse histological subcategories. Chondroid lipoma is an extremely exceptional, benign adipocytic neoplasm initially scripted by Meis and Enzinger in 1993 as a tumefaction simulating liposarcoma or myxoid chondrosarcoma [1]. As the neoplasm is composed of mature adipocytes, lipoblasts and myxochondroid stroma, chondroid lipoma may be histologically misinterpreted as an adipose tissue sarcoma, round cell liposarcoma or extra-skeletal myxoid chondrosarcoma. As malignant tumours are therapeutically managed with adjuvant radiotherapy, chemotherapy, and extensive surgical resection wherein benign tumours are appropriately addressed by marginal resection, cogent distinction between benign lipomatous neoplasms from diverse malignant neoplasms is necessitated to circumvent unnecessary therapeutic intervention and associated adverse outcomes. The essentially benign chondroid lipoma does not mandate expansive surgical extermination, adjunctive radiotherapy, or chemotherapy.

\section{Disease Characteristics}

Chondroid lipoma is a neoplasm commonly emerging within middle aged adults, preponderantly within the third decade. Median age of disease emergence is 36 years, and the condition can arise from 14 years to 70 years [2]. The tumefaction is accompanied by significant female predominance with a female to male proportion of 4:1 [2,3]. The exceptional, benign chondroid lipoma can arise at pertinent locations as proximal extremities or limb girdle. Nevertheless, sites such as trunk, head and neck, oral cavity, breast and abdominal cavity may be implicated. Majority of lesions incriminate the subcutaneous tissue, superficial muscular fascia, or skeletal muscle [2,3]. On cytogenetic analysis, the neoplasm is associated with chromosomal translocation $(11 ; 16)$, akin to a hibernoma [2] Tumefaction can represent as a sitespecific, gradually progressive, painless, hard, mobile nodule. The condition is associated with a non-aggressive clinical course $[2,3]$.

Histological Elucidation On gross examination, a lobulated, encapsulated, firm to hard tumor nodule of variable magnitude is delineated. Tumor magnitude extends up to 11 centimeters with a median diameter of up to 4 centimeters. Cut surface is lobulated, yellowish or grey/white with glistening areas [3,4]. On fine needle aspiration cytology, mature lipocytes and lipoblast- like cells are disseminated within a chondromyxoid matrix [3]. On microscopy, the well circumscribed and lobulated tumor is composed of mature adipose tissue and lipoblast- like cells with an encompassing myxoid and chondroid matrix $[3,4]$. Tumefaction is composed of nests and cords of miniature to medium-sized, multi-vacuolated cells simulating lipoblasts, chondroblasts or cells of a hibernoma. Enveloping stroma is prominently chondromyxoid with variable quantities of mature adipose tissue.

Foci of metaplastic bone may be exceptionally discerned $[3,4]$. The well limited, encapsulated neoplasm consists of mature lipoid cells amalgamated within the tumor perimeter admixed with disseminated chondroid cells [4]. Generally, clusters, strands or sheets of vacuolated, eosinophilic cells imbued with glycogen and lipid droplets are observed. Encompassing stroma is variable and composed of mature adipose tissue and a partially fibrinous or hyalinized, significantly myxoid matrix [3,4]. Neoplasm exhibits multi-vacuolated, eosinophilic cells configuring strands, nests and sheets variably admixed with mature adipocytes along with a circumscribing myxoid or chondroid -like stroma $[3,4]$.

Tumor cells of chondroid lipoma simulate lipoblasts, chondroblasts and cells of brown fat or hibernoma wherein tumor cell nuclei display complex nuclear outlines. Enveloping stroma 


\section{Cancer Therapy \& Oncology International Journal}

usually delineates prominent vasculature with variable thickness of vessel walls. Foci of hemorrhage, fibrosis and hemosiderin pigment deposition are observed along with zones of eosinophilic, fibrinous matrix. Cellular and nuclear pleomorphism, atypia and mitotic activity are minimal to absent. The neoplasm is devoid of mature hyaline cartilage [3,4]. Multi-vacuolated cells are incorporated with mature fat and glycogen which can be highlighted by Oil Red 0 and periodic acid Schiff's (PAS) stain [3,4]. On ultrastructural examination, abundant intracytoplasmic lipid and glycogen vacuoles are accompanied by numerous pinocytotic vesicles, a feature which is characteristic of mature white adipocytes. Tumor cell zones may recapitulate embryonal fat or embryonal cartilage. Cells depict knob- like protrusions of the cell membrane which are impacted with granular, amorphous, and fibrillary material. Mitochondria or lysosomes are insignificant $[3,4]$ (Figures 1-8).

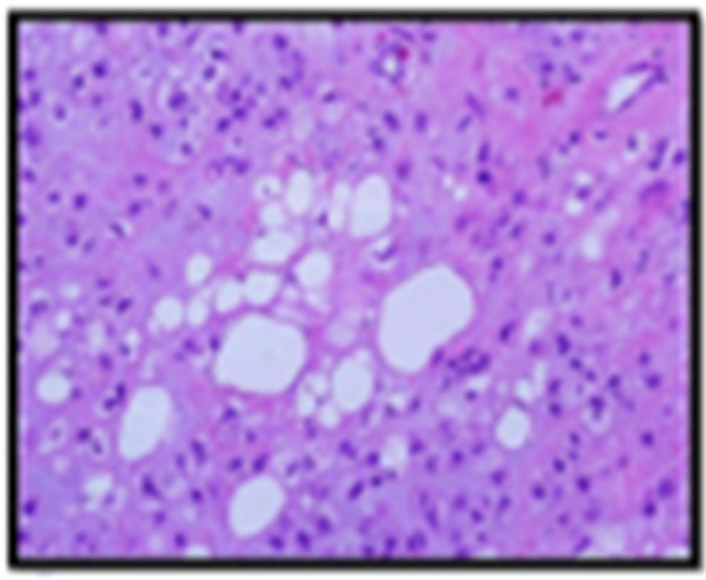

Figure 1: Chondroid lipoma enunciating clusters of mature adipose tissue cells admixed with multi-vacuolated cells with eosinophilic cytoplasm and enveloping chondromyxoid stroma.

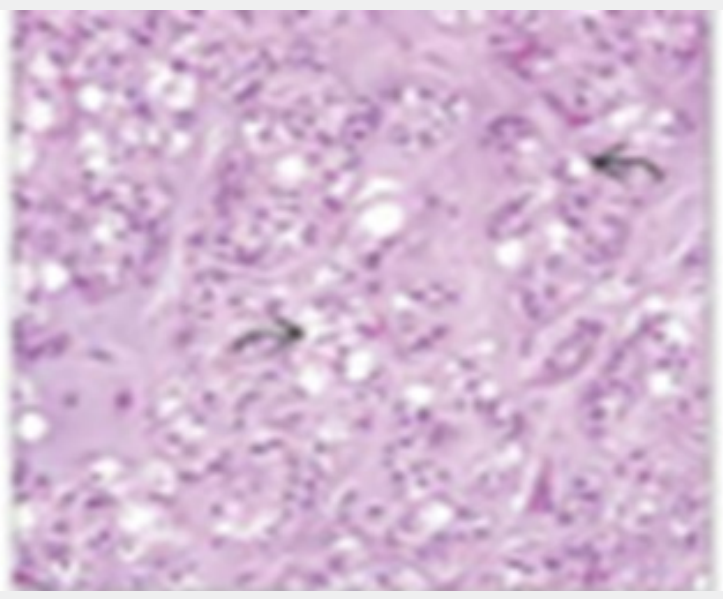

Figure 2: Chondroid lipoma exemplifying mature adipose tissue cells intermixed with multi-vacuolated cells with eosinophilic cytoplasm and an enveloping chondromyxoid stroma.

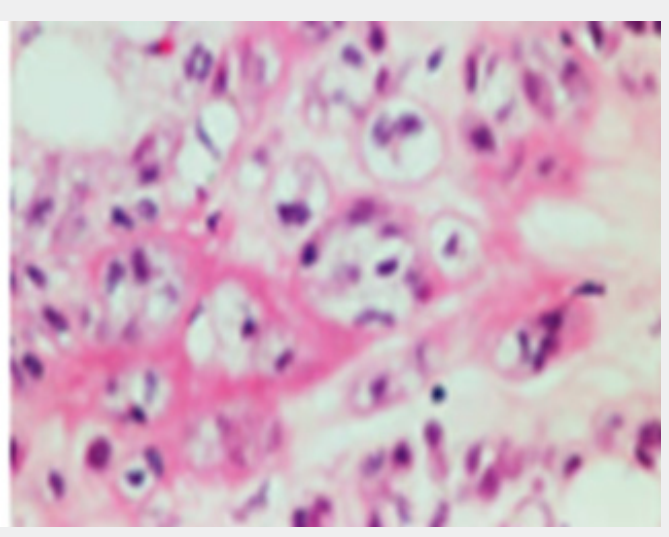

Figure 3: Chondroid lipoma demonstrating multi-vacuolated cells with eosinophilic cytoplasm, nests of mature adipose tissue cells and a surrounding chondromyxoid stroma.

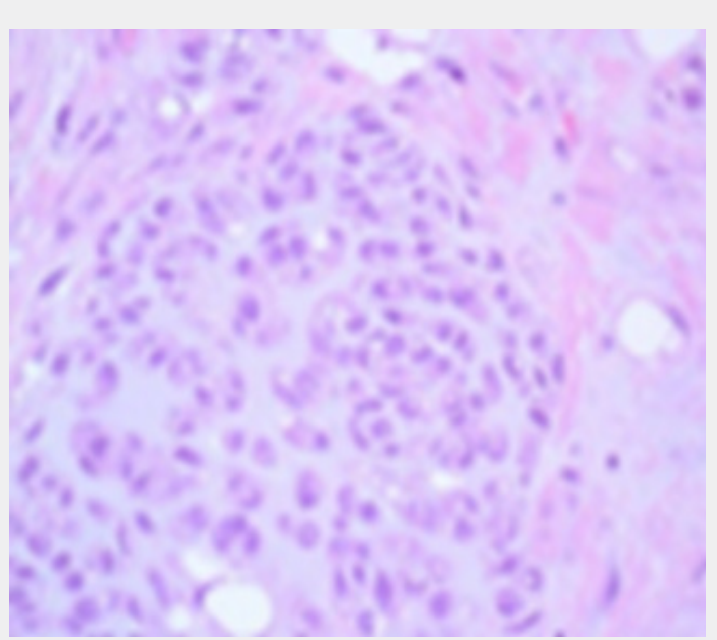

Figure 4: Chondroid lipoma delineating multi-vacuolated cells with eosinophilic cytoplasm, few mature adipose tissue cells and an encompassing chondromyxoid stroma.

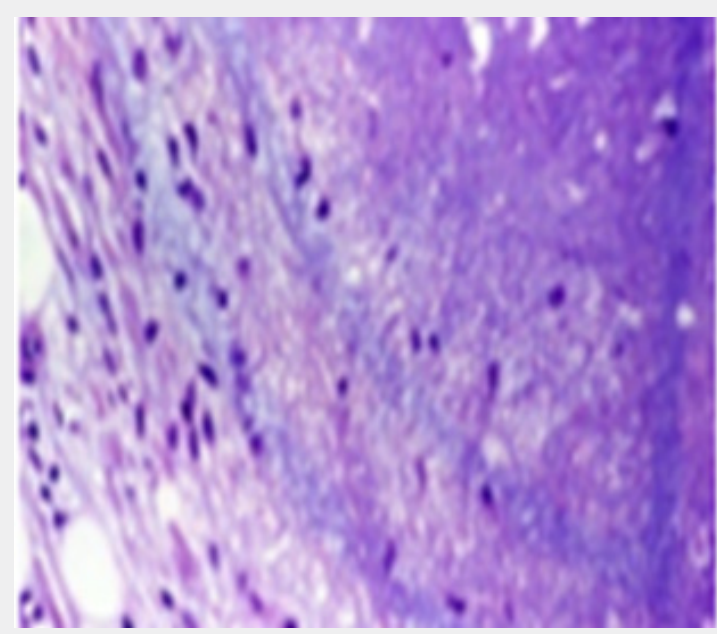

Figure 5: Chondroid lipoma exhibiting multi-vacuolated cells with eosinophilic cytoplasm, a surrounding myxochondroid stroma and few mature adipose tissue cells. 


\section{Cancer Therapy \& Oncology International Journal}

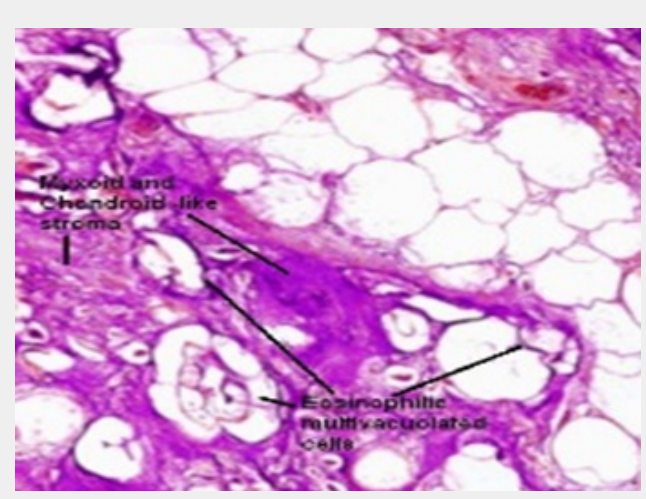

Figure 6: Chondroid lipoma depicting clusters of mature adipose tissue cells admixed with enlarged cells with eosinophilic cytoplasm and uniform nuclei and a circumscribing myxoid and chondroid stroma [14].

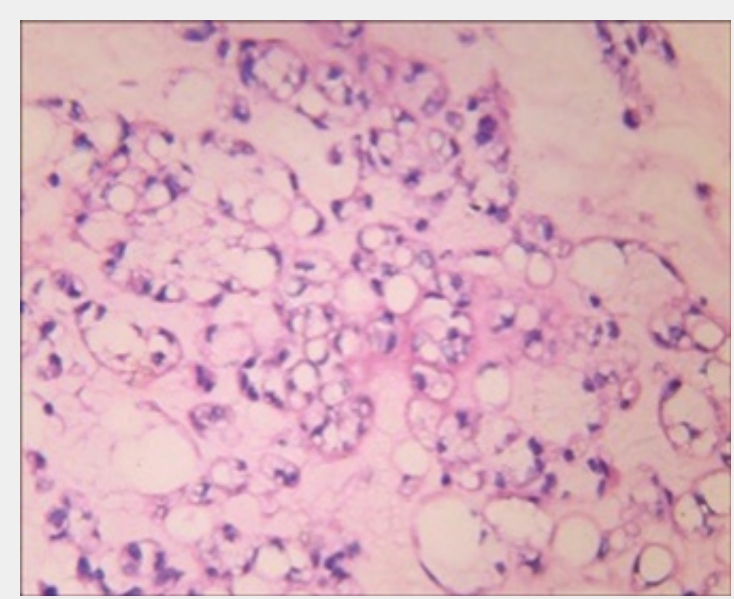

Figure 7: Chondroid lipoma demonstrating foci of multivacuolated cells with abundant, eosinophilic cytoplasm admixed with mature adipose tissue cells and an intermingled myxochondroid stroma [15].

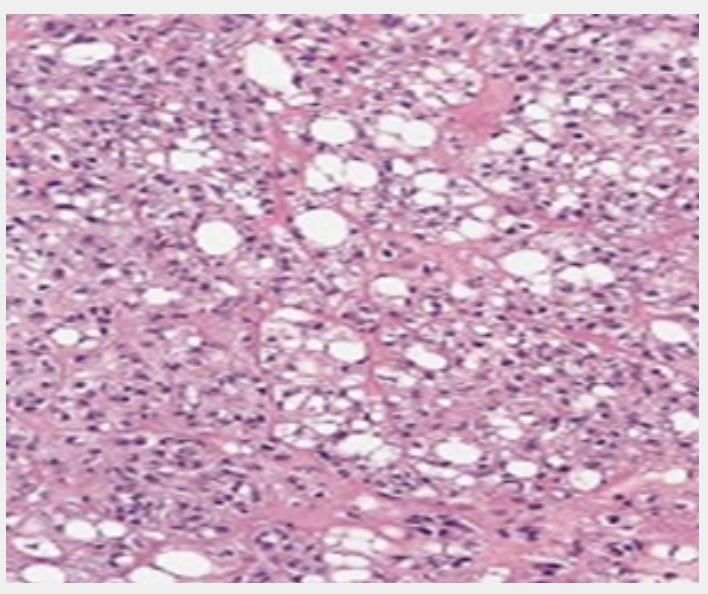

Figure 8: Chondroid lipoma depicting foci of mature white adipose tissue cells intermingled with multi-vacuolated chondroblast-like with abundant, eosinophilic cytoplasm and circumscribing myxoid matrix [15].

\section{Immune Histochemical Elucidation}

Chondroid lipoma is immune reactive to vimentin and S100 protein. Mature adipocytes are intensely immune reactive to S100 protein, in contrast to minimally reactive lipoblasts. Intense immune reactivity to cyclin $\mathrm{D}_{1}$ and variable, focal immune reactivity to CD68 and cytokeratin is observed. Circumscribed tumor cell aggregates are immune reactive to collagen IV which highlights the basement membrane. MIB $_{1}$ proliferation index is minimal and generally below $<1 \%$. Glycogen is highlighted by periodic acid Schiff's (PAS) stain whereas chondroitin sulphate is stained by alcian blue or toluidine blue $[5,6]$. The neoplasm is immune non-reactive to epithelial membrane antigen (EMA), human melanoma black 45 (HMB-45) antigen and smooth muscle actin (SMA). Tumor cells are immune non-reactive to cyclindependent kinase $4\left(\mathrm{CDK}_{4}\right)$, mouse double minute homolog 2 $\mathrm{MDM}_{2}$, monoclonal antibody to Ki-67 (MIB $)$ and transcription factor $\operatorname{SOX}_{9}(6)$. Cytogenetic analysis demonstrates a balanced chromosomal translocation $t(11 ; 16)$ (q13; p12-13) [5,6].

\section{Differential Diagnosis}

Benign or malignant adipose tissue neoplasms with chondroosseous differentiation such as lipoma, liposarcoma along with tumours with myxoid component as myxoid liposarcoma or extraskeletal myxoid chondrosarcoma are tumefaction which require demarcation from chondroid lipoma [6]. Chondroid lipoma histologically recapitulates liposarcoma, chondrosarcoma, myxoid liposarcoma and osteosarcoma. Chondroid lipoma necessitates a segregation from neoplasms such as Myxoid liposarcoma which is devoid of prominent cords and aggregates of tumor cells and displays a characteristic plexiform, vascular network. The extensively cellular neoplasm is composed of true lipoblasts and appears at diverse sites. Upon magnetic resonance imaging (MRI), myxoid liposarcoma depicts a reticulated, linear pattern on account of miniature quantities of mature adipose tissue or a distinctive, cyst -like appearance [6,7]. extra-skeletal myxoid chondrosarcoma is an infiltrative, lobulated neoplasm traversed with prominent fibrous tissue septa and circumscribed by an attenuated, peripheral capsule. Mature adipocytes and lipoblasts are usually absent. Constituent, spheroidal chondroblasts are typically devoid of intracytoplasmic vacuoles. Mitotic figures and focal tumor necrosis are frequently observed $[6,7]$.

Extra-skeletal chondroma is a neoplasm commonly located within distal extremities. Tumefaction demonstrates foci of true, mature hyaline cartilage and disseminated multinucleated giant cells. Mature adipose tissue cells and intracytoplasmic lipid vacuoles are usually absent. mixed tumor is a neoplasm devoid of lipoblasts. Foci of epithelial differentiation are observed. The neoplasm is immune reactive to keratin [6,7]. Osteosarcoma demonstrates tumor permeation with bone erosion and cortical bone destruction. Tumor cells are pleomorphic and hyperchromatic with diverse morphology as epithelioid, plasmacytoid, spindle-shaped, miniature spherical cells, clear 


\section{Cancer Therapy \& Oncology International Journal}

cells and tumor giant cells. Discernible foci of neoplastic osteoid are diagnostic of the malignancy $[7,8]$. lipoma with chondroid metaplasia demonstrates foci of true cartilage. Lipoma is composed of mature white adipose tissue cell aggregates, uniform cytoplasmic vacuoles, tumor parenchyma traversed by fibrous tissue septa and lack of cellular atypia or mitosis $[7,8]$.

\section{Investigative Assay}

Chondroid lipoma is associated with uncommon, pathognomonic radiographic features. Thereby, demarcation from associated malignant neoplasia can be challenging. Chondroid lipoma can be subjected to plain radiography, ultrasonography, and magnetic resonance imaging (MRI) for appropriate assessment. Plain x-ray demonstrates a soft tissue tumefaction associated with or devoid of calcification [7,8]. Upon magnetic resonance imaging (MRI), a well-defined, lobulated tumor mass is discerned. Upon $\mathrm{T}_{1}$ weighted imaging, the heterogeneous mass demonstrates an enhanced signal intensity indicative of mature adipose tissue, in contrast to circumscribing skeletal muscle $[7,8]$. Upon $\mathrm{T}_{2}$ weighted imaging, fibrous tissue septa traversing the tumor parenchyma exhibit a minimal signal intensity. Periphery of tumor nodule depicts enhanced signal intensity upon $\mathrm{T}_{1}$ and $\mathrm{T}_{2}$ weighted imaging, in contrast to encompassing skeletal muscle. Upon MRI, a "fat ring" sign is discerned which suggests a diagnosis of chondroid lipoma $[7,8]$. Upon $\mathrm{T}_{2}$ weighted fatsuppressed imaging, signal intensity of tumor mass is suppressed. A non-uniform fat saturation is discerned upon $\mathrm{T}_{2}$ weighted fat- saturated imaging, thereby suggesting the presence of mature adipose tissue $[8,9]$. Gadolinium- enhanced $\mathrm{T}_{1}$ weighted imaging depicts a nodule with enhanced signal intensity. Delayed enhancement of signal intensity following administration of gadolinium-contrast indicates the presence of chondroid tissue. Post contrast imaging delineates an intense signal enhancement, especially at the tumor periphery [8,9]. Aforesaid imaging features are indicative of chondroid lipoma. Quantifiably variable components of mature adipose and chondroid tissue along with admixed myxoid matrix exemplify heterogeneous, nonspecific signal intensities upon MRI. Thus, minimal signal intensity upon $\mathrm{T}_{1}$ weighted imaging and enhanced signal intensity upon $\mathrm{T}_{2}$ weighted imaging is exemplified. Aforesaid pattern of signal intensities is indicative of chondroid matrix $[8,9]$. Additionally, reticulated strands of elevated signal intensity upon $\mathrm{T}_{1}$ weighted imaging which are suppressed on short-tau inversion recovery (STIR) imaging indicate the concurrence of mature adipose tissue. The well demarcated, lobulated tumefaction demonstrates a centroidal enhancement of signal intensities with configuration of rings and arcs which is indicative of a cartilaginous tumor. Centric segment of tumefaction is predominantly composed of chondroid component and peripheral tumor segment is predominantly constituted by mature adipose tissue $[8,9]$.

Adoption of diffusion- weighted MRI is beneficial in evaluating soft tissue neoplasms as the apparent diffusion coefficient of benign tumours is significantly elevated, in contrast to malignant tumours. Tissue diffusion is estimated by equivalence between tumor cellularity and stromal matrix. Thus, diffusionweighted MRI is advantageous in segregating chondroid lipoma from sarcoma $[8,9]$. A fluoro-deoxy glucose positron emission tomography (FDG-PET) employed for assessing benign or low grade tumours such as chondroid lipoma demonstrate reduced standard uptake value $\left(\mathrm{SUV}_{\max }\right)$. Nevertheless, radiography and ultrasonography cannot suitably differentiate between chondroid lipoma and malignant soft tissue sarcomas $[8,9]$.

\section{Therapeutic Options}

Tumefaction can be subjected to marginal resection or a comprehensive surgical extermination. Cogent surgical excision is curative. The benign chondroid lipoma is devoid of localized tumor reoccurrence or distant metastasis following adequate surgical eradication. Malignant metamorphosis is undocumented. Precise tumor discernment is essential to circumvent excessive or radical therapy $[8,9]$.

\section{References}

1. Meis JM, Enzinger FM (1993) Chondroid lipoma- a unique tumour simulating liposarcoma and myxoid chondrosarcoma. Am J Surg Pathol 17(11): 1103-1112.

2. Katsuyama Y, Shirai T, Ryu Terauchi, Shinji Tsuchida, Naoki Mizoshiri et al (2018) Chondroid lipoma of the neck- a case report" BMC Res Notes 11(1): 415.

3. Yildiz AE, Aydingoz U, Cenk Sökmensüer, Muşturay Karçaaltıncaba et al (2015) Intramuscular chondroid lipoma- magnetic resonance imaging diagnosis by "fat ring sign" Balkan Med J 32(1): 107-110.

4. Tannenbaum M, Colucci PG, Michael Baad, Alain C Borczuk, Shaun A Steigman et al" (2020) Chondroid lipoma- multimodality imaging in a 9-year-old female" Skeletal Radiol 49(1): 161-169.

5. Chao H, Wenlai G, Wenrui Qu, Zhe Zhu, Rui Li et al" (2019) Characteristics of chondroid lipoma- a case report and literature review" Medicine 98(19): e15587.

6. Hoch B, Hermann G (2008) Ossifying chondroid lipoma" Skeletal Radiol 37(5): 475-80.

7. Hwang HS, Lee WJ (2008) Chondrolipoma in the pelvic cavity- a case report Korean J Radiol 9(6):563-567.

8. Jung HR, Park WH (2007) Intestinal chondrolipoma-uncommon cause of bowel obstruction" J Pediatr Surg 42(10): E21-E23.

9. Thway K, Flora RS (2012) Chondroid lipoma - an update and review" Ann Diagn Pathol 16(3): 230-234. 
This work is licensed under Creative Commons Attribution 4.0 License

DOI:10.19080/CTOIJ.2020.17.555971
Your next submission with Juniper Publishers will reach you the below assets

- Quality Editorial service

- Swift Peer Review

- Reprints availability

- E-prints Service

- Manuscript Podcast for convenient understanding

- Global attainment for your research

- Manuscript accessibility in different formats

( Pdf, E-pub, Full Text, Audio)

- Unceasing customer service

Track the below URL for one-step submission https://juniperpublishers.com/online-submission.php 